\title{
Optimization of Oil Pipe Arrangement with Conditions of Compensation and Different Unit Cost
}

\author{
Dongping Wei ${ }^{1, a}$, Tianli Lei ${ }^{2^{*}, b}$, Lv Peng $^{3, c}$ \\ ${ }^{1}$ School of Management, Shanghai University, Shanghai, 200444, China \\ ${ }^{2}$ Math and Physics Department, Shenzhen Polytechnic, Shenzhen, 518055, China; \\ ${ }^{3}$ China Mobile Group Guangdong Co.,Ltd.Dongguan Branch,Dongguan,523001,China \\ a dreamoffice@163.com, ${ }^{\mathrm{b}}$ |t|@ szpt.edu.cn, ${ }^{\mathrm{c}} 28952742 @ q q . c o m$
}

\begin{abstract}
Keywords: Oil Pipe Arrangement Fermat Problem.
Abstract. It is usually to see that oil pipelines usually pass through suburbs areas and urban areas. And it is necessary to pay compensation to private properties owners when the pipelines occupy some private properties. Meanwhile, the unit costs of different section pipelines are different because the production capacities of refineries are different. Hence, the optimal scheme of oil pipe arrangement is very complicated in such case. Hence, the oil pipe arrangement scheme with conditions of compensation and different unit cost of the oil pipelines is proposed to study the complicated case. The simulation shows that model can easily obtain the optimal scheme to construct oil pipe when given different compensation and different unit cost of the oil pipelines.
\end{abstract}

\section{Introduction}

An oilfield company plans to build two refineries on the one side of railway, and built a station in the railway to transport oil products as shown in Fig.1. The problems are complicated in some case. For instance, Moura A V reported that Brazilian Petrobras faces a very difficult over-constrained planning challenge: how to operate a large pipeline network in order to adequately transport oil derivatives and biofuels from refineries to local markets. [1]. Wei, D. and Lei, T. converted the problem of optimization gas or oil pipelines arrangement into Fermat problem of "Two points and One Line" [2]. In fact, Oil pipelines usually pass through suburbs areas and urban areas. Wei, D. and Lei, T. firstly study the simplest problem that the oil company does not need to pay the private properties owners when the pipeline occupies some private properties [3]. OAPSU-Model and OAPDU-Model is proposed to study the oil pipe arrangement scheme without compensation to private properties owners. However, it is usually to see that the oil company should pay the private properties owners when the pipeline occupies some private properties. Hence, it is quite necessary to study more complicated conditions that the company should pay compensation to private properties owners in urban or suburbs areas. Then the oil pipe arrangement scheme with compensation conditions (OPASCC-Model) is proposed Wei, D. and Lei, T [4]. Although OPASCC-Model can solve the problem better, it didn't consider that different section oil pipe has different unit cost because the production capacities of refineries are different. Hence, it is necessary to study oil pipe arrangement scheme with more complicated conditions including compensation in urban areas and different unit cost of different oil pipe section.

\section{Notations}

$k_{1}$ : The unit cost of pipelines $A P$

$k_{2}$ : The unit cost of pipelines $P F$

$k_{3}$ : the unit cost of pipelines $B F$

$k_{4}$ :The unit cost of pipelines $P E$ 
$k_{3}$ : The unit cost of pipelines in urban areas

$F:$ The total cost of pipelines

$a$ : The vertical distance from refinery A to the railway

$b:$ The vertical distance from refinery B to the railway

$l$ : The horizontal distance between refinery $\mathrm{A}$ and $\mathrm{B}$

$p$ : The Fermat point

\section{Methodologies}

\section{Oil Pipe Arrangement Scheme without Compensation to private properties owners}

The positions of A, B refineries and railway can be shown in Fig.1. Oil pipelines usually pass through suburbs areas and urban areas. The simplest problem is the oil company does not need to pay the private properties owners when the pipeline occupies some private properties. It is easy to see from Fig. 1 that $P E$ is the shared pipeline. $A P$ and $P B$ are non-shared pipeline. Wei, D. and Lei, T. proposed OAPSU-Model and OAPDU-Model to study the oil pipe arrangement scheme without compensation to private properties owners. OAPSU-Model is assumed that the unit cost of shared or non - shared pipeline is the same. OAPDU-Model is assumed that the unit cost of shared or non shared pipeline different is different. They found that OAPSU-Model is the special case of OAPDU-Model when $k_{1}=k_{2}$. Tab. 1 shows the lowest cost of whole pipelines $F_{\text {min }}$ with OAPSU-Model. Tab. 2 shows the lowest cost of whole pipelines $F_{\min }$ with OAPDU-Model.

\begin{tabular}{|l|l|l|l|}
\hline The relations of $l, a$ and $b$ & $0<l<\sqrt{3}(b-a)$ & $\sqrt{3}(b-a) \leq l \leq \sqrt{3}(b+a)$ & $l>\sqrt{3}(a+b$ \\
\hline$F_{\text {min }}$ OAPSU-Model & {$\left[a+\sqrt{l^{2}+(a-b)^{2}}\right] k$} & $\frac{a+b+\sqrt{3} l}{2} \cdot k$ & $\sqrt{l^{2}+(a-b}$ \\
\hline
\end{tabular}

Tab.1 $\quad F_{\min }$ with OAPSU-Model

\begin{tabular}{|l|l|l|l|}
\hline $\begin{array}{l}\text { The relations } \\
\text { of } l, a \text { and } b\end{array}$ & $0 \leq l \leq \frac{(b-a) \sqrt{4 k_{1}{ }^{2}-k_{2}{ }^{2}}}{k_{2}}$ & $\frac{(b-a) \sqrt{4 k_{1}{ }^{2}-k_{2}{ }^{2}}}{k_{2}} \leq l \leq \frac{(a+b) \sqrt{4 k_{1}{ }^{2}-k_{2}{ }^{2}}}{k_{2}}$ & $l>\frac{\sqrt{4 k_{1}^{2}-k_{2}^{2}}}{k_{1}}(a+b)$ \\
\hline $\begin{array}{l}F_{\min } \\
\text { OAPDU-Model }\end{array}$ & $k_{1} \sqrt{l^{2}+(a-b)^{2}}+k_{2} a$ & $\frac{1}{2}(a+b) k_{2}+\frac{\sqrt{4 k_{1}^{2}-k_{2}{ }^{2}}}{2} l$ & $k_{1} \sqrt{l^{2}+(a+b)^{2}}$ \\
\hline
\end{tabular}

Tab.2 $\quad F_{\text {min }}$ with OAPDU-Model 


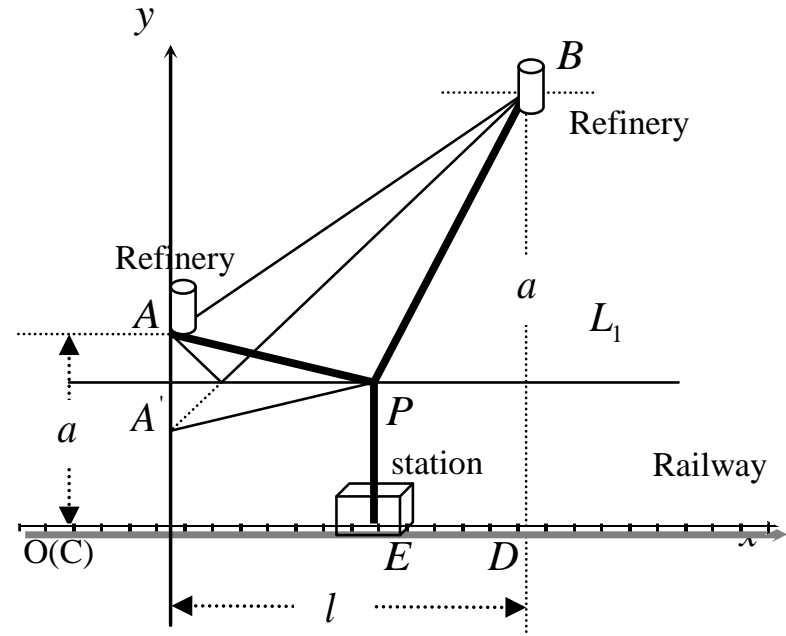

Fig.1 The arrangement of pipelines without compensation to private properties owners

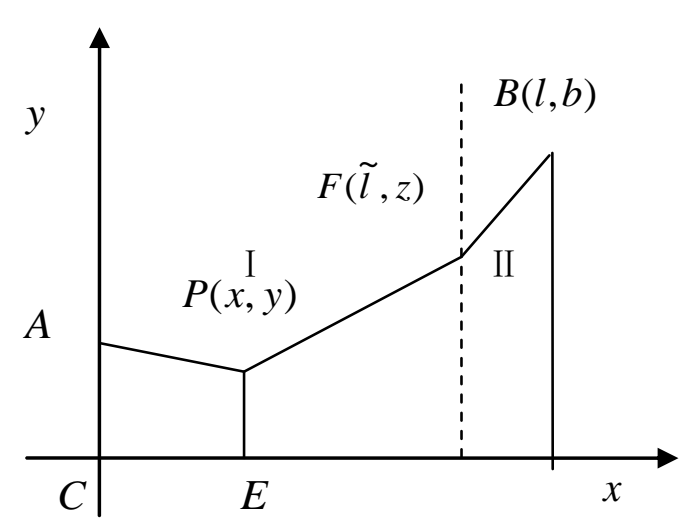

Fig.2 The arrangement of pipelines with compensation to private properties owners

\section{Oil Pipe Arrangement Scheme with Compensation to private properties owners}

\section{Oil Pipe with the same unit cost}

Oil pipelines usually pass through suburbs areas and urban areas. Fig. 2 shows that area I is the suburbs and area II is the urban. There are many private properties in urban areas. However, OAPSU-Model and OAPDU-Model assumed that the oil company does not need to pay the private properties owners when the pipeline occupies some private properties. It is usually to see that the oil company should pay the private properties owners when the pipeline occupies some private properties. Hence, it is quite necessary to study more complicated conditions that the company should pay compensation to private properties owners in urban or suburbs areas. Wei, D. and Lei, T. proposed the oil pipe arrangement scheme with compensation conditions (OPASCC-Model) as following to study the complicated problem.

$$
\begin{aligned}
& \min F=k_{1} \sqrt{\tilde{l}^{2}+(2 y-a-z)^{2}}+k_{1} y+k_{3} \sqrt{5^{2}+(b-z)^{2}} \\
& \text { s.t } 0 \leq y \leq a \\
& \quad 0 \leq z \leq 8
\end{aligned}
$$

The OPASCC-Model shows that compensation cost can affect the pipeline arrangement very sensitively. The model can obtain the optimal scheme to construct oil pipe when given different compensation cost. The OPASCC-Model shows that pipe arrangement in Fig.1 change to $A P, P E, P F, F B$ shown in Fig.2. However, OPASCC-Model also assumed that the unit cost of pipeline $A P, P E, P F, F B$ is the same.

\section{Different section oil pipe with unit cost}

In fact, the unit cost of pipeline $A P, P E, P F, F B$ is different because the production capacity of $A$ and $B$ refineries are different. Then OPASCC-Model is not suitable to obtain the optimal scheme for arranging the oil pipe in such case. So it is necessary to study oil pipe arrangement scheme with more complicated conditions including compensation in urban areas and different unit cost of different oil pipe section. $k_{1}$ is denoted as the unit cost of $A P . k_{2}$ is denoted as the unit cost of $P F . k_{3}$ is denoted as the unit cost of $B F . \quad k_{4}$ is denoted as the unit cost of $P E$. Therefore, oil pipe 
arrangement scheme with conditions compensation and different unit cost ( OPASCCDUC-Model)can be described as following

$$
\begin{aligned}
& \min F=k_{1} \sqrt{x^{2}+(y-a)^{2}}+k_{2} \sqrt{(15-x)+(y-z)^{2}}+k_{3} \sqrt{5^{2}+(8-z)^{2}}+k_{4} y \\
& \text { s.t. } \quad 0 \leq x \leq 15 \\
& \quad 0 \leq y \leq a \\
& \quad 0 \leq z \leq 8
\end{aligned}
$$

When $k_{1}=5.6, k_{2}=6, k_{3}=27, k_{4}=7.2$, it is easy to obtain the optimal solution by the method of multiple-variable calculus [5] that $x=6.7424, y=0.1327, z=7.2659, F=249.4422$. Similarly, it is not difficult to calculate the optimal solution of OPASCCDUC-Model by MATLAB program [6] when given three different compensation cost shown in Tab.3.

\begin{tabular}{lccccc}
\hline & The unit cost of pipeline & \multicolumn{2}{c}{ The Fermat point $\mathrm{p}$} & Point & The total cost \\
\hline$k_{3}$ & $\mathrm{y}$ & $\mathrm{x}$ & $\mathrm{F}$ & $F_{\min }$ \\
Firm 1 & 27 & 0.1327 & 6.7424 & 7.2659 & 249.4422 \\
Firm 2 & 30 & 0.1667 & 6.6952 & 7.3406 & 264.586 \\
Firm 3 & 26 & 0.1195 & 6.7606 & 7.2370 & 244.3865 \\
\hline
\end{tabular}

Tab.3 The optimal pipeline arrangement with different compensation cost and different unit cost

Note: $k_{3}$ is the unit cost of Pipelines in urban areas+ compensation cost According to Table 3, it can design three options oil pipeline arrangement plan as following:

1) Plan I: In the case of Firm 1 , the Fermat point is $P(6.7424,0.1327)$. The turning point is $F(15,7.2659)$.The station is located at $E(6.7424,0)$.The pipelines of $P A=8.3157 \mathrm{~km}$, $P E=0.1327 \mathrm{~km}, P F=10.9119 \mathrm{~km}, \quad F B=5.0536 \mathrm{~km}$. The total cost of pipelines is $2,494,422$ RMB.

2) Plan II: In the case of Firm 2, the Fermat point is $P(6.6952,0.1667)$. The turning point is $F(15,7.3406)$.The station is located at $E(6.6952,0)$.The pipelines of $P A=8.2575 \mathrm{~km}$, $P E=0.1667 \mathrm{~km}, P F=10.9743 \mathrm{~km}, \quad F B=5.0433 \mathrm{~km}$. The total cost of pipelines is 2,645,862 RMB.

3) Plan III: In the case of Firm 3, the Fermat point is $P(6.7606,0.1195)$. The turning point is $F(15,7.2370)$.The station is located at $E(6.7606,0)$.The pipelines of $P A=8.3382 \mathrm{~km}$, $P E=0.1195 \mathrm{~km}, P F=10.8879 \mathrm{~km}, \quad F B=5.0579 \mathrm{~km}$. The total cost of pipelines is $2,645,862$ RMB.

\section{Conclusions}

This paper assumes that the company should pay compensation to private properties owners in urban or suburbs areas when the pipeline occupies some private properties. It is also assumed that the unit cost of different section pipeline $A P, P E, P F, F B$ is different because the production capacities of refineries are different. Then the oil pipe arrangement scheme with conditions of compensation and different unit cost (OPASCCDUC-Model) is proposed to study the complicated case. Finally, the simulation of OPASCCDUC-Model gives three different optimal plans to construct oil pipe when given three different compensation cost. 


\section{Acknowledgements}

This work is supported by Social Science Foundation of Guangdong (Grant no. GD14XGL03) and Youth Innovation fund of Shenzhen polytechnic(Grant No. 601422K35018), Social Science Project of Shenzhen (Grant no. 125C09). And the authors would like to thank the editor and an anonymous referee for their helpful comments on the manuscript.

\section{References}

[1] Moura, Arnaldo V. Planning and scheduling the operation of a very large oil pipeline network. Principles and Practice of Constraint Programming, Springer Berlin Heidelberg, 2008.

[2] Kuhn H W, A note on Fermat's problem, Mathematical programming, 1973, 4(1): 98-107.

[3] Dongping, wei, Hongya,Zhao,Tianli,Lei, Optimization in Oil Pipelines Arrangement with Fermat Problem .2014 International Conference on Economic Management and Trade Cooperation,2014,212-217.

[4] Wei D, Zhao H, Lei T. Optimization of Oil Pipe Arrangement with Compensation Conditions. Applied Mechanics \& Materials, 2014, 568-570:1675-1679.

[5] Widder D V, Advanced calculus, Courier Dover Publications, 2012.

[6] Hanselman, Duane C., and Bruce Littlefield. Mastering matlab 7, Pearson/Prentice Hall, 2005. 